\title{
Die SwissDRG stellen neue Herausforderungen an das OP-Management
}

\author{
Martin Doser, \\ Christoph Egger Halbeis, \\ Guido Schüpfer
}

Potentielle Interessenkonflikte: keine.
Korrespondenz:

Dr. med. Martin Doser, D.E. A. A. Oberarzt

Klinik und Poliklinik

für Anästhesiologie

Inselspital

CH-3010 Bern

martin.doser@insel.ch

\section{Einleitung: SwissDRG als Heraus- forderung der nahen Zukunft}

Im Jahr 2004 wurde das Projekt «Swiss Diagnosis Related Groups» (SwissDRG) mit folgenden Zielen initiiert:

1. Ablösung der diversen Abgeltungssysteme (Tagespauschalen, Fallpauschalen, u.a.) durch Schaffung eines neuen Fallpreispauschalensystems zwecks schweizweiter Vereinheitlichung der Tarife im stationären Bereich;

2. Schaffung von Preistransparenz für stationäre Leistungen;

3. Schaffung eines kontrollierten Wettbewerbs;

4. Schaffung eines Führungsinstrumentes für Kantone und Spitäler.

Der Verein SwissDRG hat im Dezember 2005 beschlossen, das Deutsche DRG-System (G-DRG) als Grundlage für die SwissDRG zu übernehmen [1]. Dieser Artikel soll aus der Perspektive des Operationssaal-(OP-)Managements die Einführung der SwissDRG per 2009 kritisch beurteilen und den Umgang mit Risiken diskutieren.

\section{Wie funktioniert ein DRG-System?}

DRG-Systeme gehen davon aus, dass die Patientenbetreuung und der damit verbundene Ressourcenaufwand (Kosten) im wesentlichen von der Hauptdiagnose, den Nebendiagnosen und den durchgeführten Prozeduren bestimmt werden. Je nach DRG-System werden zusätzliche Verfeinerungsmethoden angewendet, um der Komplexität des individuellen Falles gerecht zu werden. Für die Codierung der Haupt- und Nebendiagnosen gilt im G-DRG-System der ICD10-Katalog; die Prozeduren werden in Deutschland nach einem spezifischen OPS-301-Katalog verschlüsselt. In der Schweiz werden in einigen Kantonen bereits All-Patient-DRG-(AP-DRG-) Systeme verwendet, für deren Codierung der ICD-10-Katalog und CHOP-9-Katalog verwendet wird. Mittels einer speziellen Software, dem sogenannten Grouper, werden Tausende von Kombinationsmöglichkeiten von Haupt- und Nebendiagnosen und Prozeduren zu einzelnen Gruppen zusammengefasst. Letztlich dient die Abbildung der von einem Leistungserbringer erbrachten Leistungen in einem DRG-System der Komplexitätsreduktion. Der Dienstleistungs- erbringer wird aufgrund der Erfassung von Leistungs- und Diagnosecodes entschädigt.

\section{Vorgehen zur Festlegung der Preise}

Im Vorfeld der Einführung eines DRG-Systems muss für die Festlegung der Tarife eine umfassende Erhebung von Fallkosten erfolgen. In der Schweiz wurde im Herbst 2005 die Erhebung der diagnosebezogenen Fallkosten lanciert. Dabei stellen rund 40 Akutspitäler aus der ganzen Schweiz die für die Berechnung von Fallpauschalen notwendigen Patienten- und Kostendaten SwissDRG zur Verfügung [2]. Bei der Erhebung der Fallkosten wird auf eine Vollkostenrechnung verzichtet. Beispielsweise dürfen Investitionskosten gar nicht und Kosten für Lehre und Forschung nur teilweise zur Kostengewichtung der Fallgruppen deklariert werden. Unklar bleibt, wie die Kosten für die Weiterbildung von Ärzten zu Fachärzten oder von Pflegepersonal abgebildet werden.

Aus den gesammelten Daten wird pro DRG ein Relativgewicht (Costweight $[\mathrm{CW}]=$ Fallschwere) berechnet. Das Relativgewicht widerspiegelt die Kostenintensität eines bestimmten Falles bezogen auf den Standardwert (mit dem Relativgewicht 1,0).

$\mathrm{Zu}$ Beginn der Einführung wird pro Spitalkategorie - und initial sicherlich auch regional eine unterschiedliche Baserate berechnet. Die Baserate ist eine für die betreffende Spitalkategorie berechnete Pauschale in Franken. Der Erlös für eine DRG-Gruppe errechnet sich somit aus $\mathrm{CW} \times$ Baserate. Je nach Kombination von Hauptund Nebendiagnosen resultieren DRG-Einteilungen mit einem höheren CW, was zu einem höheren Erlös für das Spital führt.

\section{Welche Folgen sind für Leistungs- erbringer mit chirurgischen Patienten antizipierbar?}

Die Einführung des DRG wird mit seinen neuen wirtschaftlichen Anreizsystemen die Spitäler auf den Ebenen der Spitalführung, Abteilung (Chirurgie, Anästhesie, OP) und Mitarbeiter grundlegend beeinflussen.

Diejenigen Spitäler, die ihre Prozesse dem neuen DRG-System, d.h. einem fallbasierten Vergütungssystem, noch nicht angepasst haben, 
werden eine Ressourcenverknappung erfahren [3]. Da bei der Festlegung der SwissDRG-Tarife auf eine Vollkostenrechnung verzichtet wird, nimmt der finanzielle Druck auf die Leistungserbringer $\mathrm{zu}$, und Kostenreduktionsprogramme werden zur zentralen Aufgabe [4].

Die SwissDRG werden die strategischen Entscheidungen der Spitalführung (und die zuständigen Behörden) beim Aus- oder Abbau bestimmter Dienstleistungen beeinflussen. Ausgliederungsoptionen für verschiedene Dienste werden in Betracht gezogen werden (z.B. Radiologie, Anästhesie). Patienten werden früher entlassen werden, weil eine kürzere Hospitalisationsdauer zu einem günstigeren Betriebsergebnis führt.

Die Spitalführung wird in Zukunft nicht mehr die Bettenauslastung, sondern den Deckungsbeitrag pro Bett maximieren wollen. Der Deckungsbeitrag entspricht dabei der Differenz von Erlös (definiert durch Baserate und CW) minus Kosten pro Behandlungsfall. Mit dem Projekt REKOLE $^{\circledR}$ (Revision der Kostenrechnung und der Leistungserfassung) von $\mathrm{H}+$, der Vereinigung schweizerischer Krankenhäuser, wurden die Voraussetzungen für die Spitäler geschaffen, sämtliche anfallenden Kosten auf einen Behandlungsfall auszuweisen. Somit gewinnen die internen Prozesse zunehmend an Bedeutung mit dem Ziel, die Kostenstruktur innerhalb des Behandlungsprozesses zu optimieren.

Das Augenmerk wird sich auch auf den OP richten, weil hier viel Kapital gebunden ist und hohe fixe Betriebskosten anfallen. Nach Bach betragen die Kosten im Operationsbereich etwa 25-50\% der gesamten Fallkosten [4]. Unter diesem Aspekt ist ein zentrales OP-Management von strategischer Bedeutung.

Im weiteren werden die Anforderungen an die Dokumentation und die Qualität des Kodierens und damit auch die administrativen Kosten weiter steigen. Nach der Einführung des G-DRG wurde in Deutschland teilweise eine «systematische Jagd» nach Diagnosen beobachtet [5]. Der Dokumentationsaufwand in Deutschland im Jahre 2002 wurde auf 15\% der täglichen Arbeitszeit geschätzt, eine weitere Zunahme wird erwartet. Das korrekte Codieren mittels Grouper wird zu einer zentralen Aufgabe eines jeden Spitals. Hier zeigen sich bedeutende Vorteile einer spezialisierten Codierabteilung gegenüber dem Codieren durch den Stationsarzt [6]. Daneben stellen auch eine minutiöse Leistungserfassung am Ort der Leistungserbringung, eine gut dokumentierte, elektronische Krankengeschichte und die umfassende Mitarbeiterinformation in bezug auf das neue Abgeltungssystem neue Erfolgspotentiale dar.
Die Durchführung von Produktivitätsanalysen und die Einhaltung von Kernprozessen werden zu entscheidenden Aufgaben, wozu eine gute Zusammenarbeit zwischen Spital- und Abteilungsführung erforderlich ist. Von grossem Interesse ist das Verhältnis von Produktivität und Qualität. Zur optimalen Lösung dieser Frage gewinnen klinische Behandlungspfade (clinical pathways, CP) und Standard Operating Procedures (SOPs) zunehmend an Bedeutung. Sie definieren und sichern den Standard der Patientenbehandlung (Qualität) trotz verkürzter Hospitalisationsdauer [7]. Behandlungspfade sollten für jedes Spital individuell - ausgehend von evidenzbasierter Medizin (EBM) und spitalinternen Leitlinien - entwickelt werden. Werden CP und Fallkostenkalkulation kombiniert, so entstehen effektive Instrumente, die für die Umsetzung der Anforderungen einer DRG-Einführung von grossem Nutzen sind, wie das Modell der integrierten Patientenpfade in Aarau (mipp) vermuten lässt $[8,9]$. Die detaillierte Prozesskostenrechnung hilft den Abteilungen, ihre Vollkosten auszuweisen. Die Kenntnisse der eigenen Kosten sind im Rahmen der Allokation des DRG-Erlöses innerhalb des Spitals von grosser Bedeutung. Der Verteilungskampf unter den einzelnen Leistungserbringern um die Zuteilung der gerechten Anteile des DRG-Erlöses ist vorprogrammiert.

Die Erfahrungen in Deutschland haben gezeigt, dass das DRG-System durch neue Anreizeffekte auch die Anästhesiepraxis beeinflusst. Werden beispielsweise Spitäler für geburtshilfliche Epiduralanalgesie nicht mehr entschädigt, so führt dies längerfristig zu Reduktionen dieser Dienstleistungen [10]. Auf der anderen Seite spielen Anästhesisten dann eine für die Abgeltung wichtige Rolle, wenn sie zusätzliche, neu definierte DRG-Codes für Nebendiagnosen kodieren können (z.B. Langzeitintubation). Ein inkomplett definiertes Portfolio von Codes kann deshalb zu einem Verlust an Einnahmen führen. Es ist somit zu erwarten, dass auf dem Weg des Patienten durch das Spital die wirtschaftlich interessantesten Diagnostiken und Therapien durchgeführt werden, um den Gesamterlös zu optimieren [5]. Sowohl auf ärztlicher wie auch auf pflegerischer Seite müssen in Zukunft neue Abläufe in der Erfassung von relevanten Diagnosen generiert werden. So kann zum Beispiel die Pflegediagnose Stuhlinkontinenz oder eingeschränkte Mobilität durchaus DRG-erlösrelevant sein [3]. Ist diese Diagnose im Austrittsbericht nicht ersichtlich, kann sie auch nicht codiert werden. 
Die verkürzte Hospitalisationsdauer wird eine Verlagerung in den ambulanten Bereich nach sich ziehen, wozu Investitionen nötig werden. Gleichzeitig ist die Servicequalität zu halten. Kein Spital wird sich präoperative Abklärungen für einen elektiven Eingriff auf Kosten einer verlängerten Hospitalisation mehr leisten. Der Ausbau von Prämedikationsambulatorien und ambulanten chirurgischen Zentren - in den USA seit Jahren gängig - wird die Folge sein. Insbesondere die Höhe des einzelnen DRG-Kostengewichts und der (im Vergleich zur ambulanten Therapie) relative Deckungsbeitrag werden entscheiden, ob ein Dienstleistungserbringer einen Eingriff stationär oder ambulant durchführen wird. Voraussetzung dafür ist die konsequente Umsetzung der Kostenträgerrechnung. Im Einzelfall bedeutet dies, dass der Deckungsbeitrag bei ambulanter Behandlung (Erträge aus dem TARMED interne Kosten bei ambulanter Behandlung) mit demjenigen bei stationärer Therapie (Erlös aus dem SwissDRG - interne Kosten bei stationärer Behandlung) verglichen werden muss. Dies zeigt die Bedeutung der Kalkulationsgrundlagen für die Berechnung der Kostengewichte der SwissDRG. So liegen die Operationszeiten in den Ausbildungsspitälern häufig über den Operationszeiten, die im TARMED-Katalog vorgegeben sind. Wird nun als Basis für die Kalkulationen der SwissDRG der TARMED hinzugezogen, können erhebliche Verzerrungen entstehen.

Abbildung 1

Taktische und operative Entscheidungen und Ziele. In Anlehnung an eine Abbildung im Buch OP-Management der Medizinisch Wissenschaftlichen Verlagsgesellschaft, Berlin. Mit freundlicher Genehmigung des Verlages.

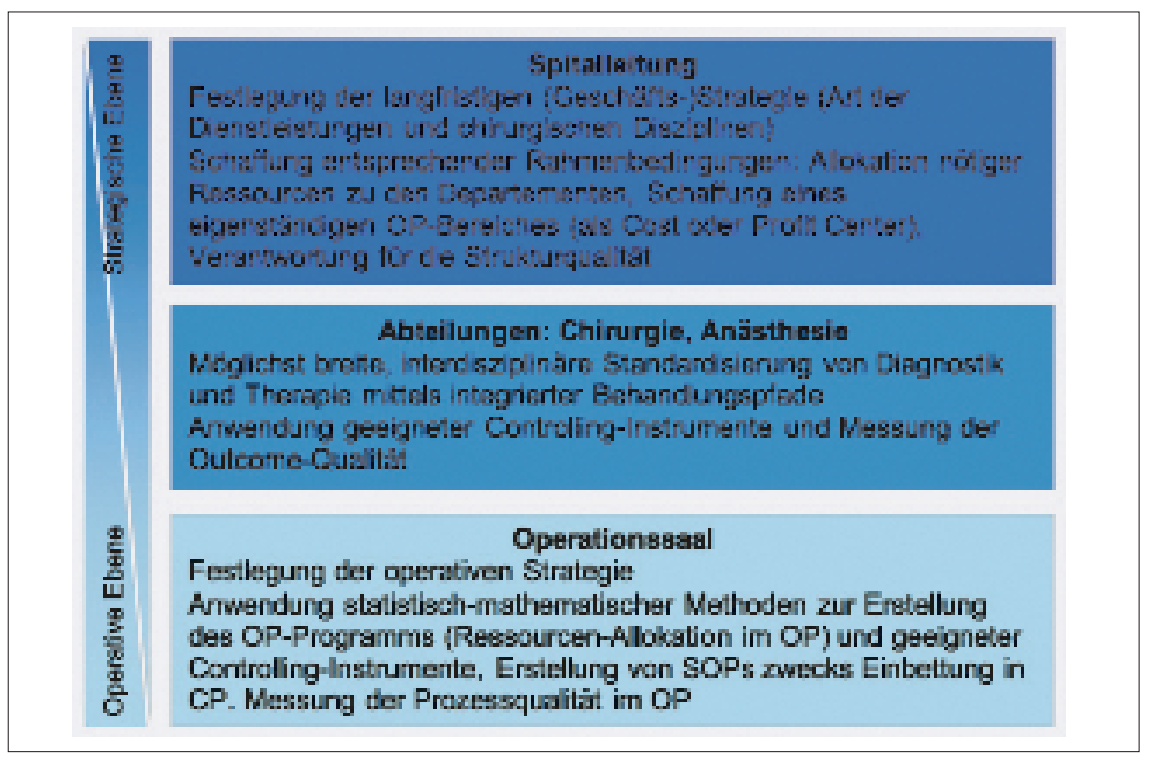

\section{Wie können sich Spitäler und OP-Manager vorbereiten?}

Die Einführung der SwissDRG wird sowohl die Spitalführung als auch Ärzte vor neue Herausforderungen stellen. Im Hinblick auf die Versorgung chirurgischer Patienten werden auf Stufe Spital, der Abteilungen und auch im Bereich des OP neue Instrumente und Abläufe zum Einsatz kommen, wobei taktische (langfristig-strategische) und operative (kurzfristig-strategische) Entscheidungen und Ziele unterschieden werden können (Abb. 1).

Im öffentlichen Spitalsystem der Schweiz werden langfristige Betriebsstrategien typischerweise auf Ebene Spitalleitung festgelegt und spielen für den OP-Manager eine eher untergeordnete Rolle. Hingegen soll der OP-Manager diejenige operative Strategie wählen, die für eine gegebene Anzahl von Operationen und mit einem gegebenen Qualitätsniveau (die zu definieren sind) die Gesamtkosten (Personal- und Sachkosten) minimiert bzw. die Produktivität der OP-Abteilung (Relation zwischen eingesetzten Mitteln und Erlösen) am Operationstag maximiert.

\section{Spitalleitung und langfristige strategische Entscheidungen}

Die sich abzeichnenden Veränderungen zwingen Spitalleitungen dazu, ihre betriebswirtschaftlichen Fähigkeiten und Kompetenzen anzupassen und mehr denn je nach ökonomischen Prinzipien auszurichten. Dies wird organisatorisch-strukturelle Reformen, Investitionen in zusätzliche Informationstechnologie und die Integration von neuen Methoden erfordern. So werden die Betriebe sich gezwungen sehen, leistungsfähige, IT-basierte Mess- und Dokumentationsinstrumente einzuführen, um effizienter Diagnosen erfassen und prozessorientierte und fallspezifische Kosteninformationen generieren zu können. Diese Zunahmen an verwaltungsund führungstechnischem Aufwand werden diametral entgegengesetzt stehen zum Ziel, den administrativen Aufwand pro Fall möglichst zu reduzieren. Für den einzelnen Spitalbetrieb wird sich neu die Frage stellen, ob gewisse Dienstleistungen und Abteilungen abgebaut, ausgelagert oder andernfalls ausgebaut werden. Grosse Betriebe (economies of scale) und spezialisierte Betriebe (center of excellence) werden zu bevorzugten Spitalmodellen. Das kleine Regionalspital mit seinem breiten Angebot an Dienstleistungen wird zum Auslaufmodell gehören. 


\section{Standard Operating Procedures (SOP) und Klinische Behandlungspfade}

In Deutschland haben einige Krankenhäuser unter dem durch die Einführung des DRG-Abgeltungssystems entstandenen Kostendruck im stationären Bereich gute Erfahrungen gemacht mit einer Standardisierung von Diagnostik, Behandlung durch Einführung von klinischen (Behandlungs-)Pfaden (CPs) und standardisierten Abläufen (SOPs). Bauer et al. haben in einer Übersicht die zur Prozessanalyse und Reorganisation von Abläufen notwendigen Schritte dargestellt und anhand eines Beispiels an ihrer Klinik in Kiel die Implementierung eines Anästhesie-SOPs erläutert [3]. Der grosse Vorteil von SOPs liegt darin, dass durch die Schaffung von Prozess-, Leistungs- und Kostentransparenz Kommunikations-, Leistungs- und Finanzplanungshilfen entstehen. Dies mündet in einer Reduktion dienstleisterseitig bedingter Variabilität in der Behandlung von Patienten und somit auch in einer Steigerung der Behandlungsqualität.

Namhafte Erfahrungen mit CPs und SOPs wurden aber auch bereits in der Schweiz gesammelt. Wie bereits schon erwähnt, kann als bekanntestes Beispiel an dieser Stelle die langjährige Erfahrung des Kantonsspitals Aarau mit der Schaffung von integrierten Behandlungspfaden (mipp) erwähnt werden [9].

\section{OP-Effizienz}

Dem OP als eine der kapitalintensivsten Einrichtungen des Spitals wird mehr noch als bisher Bedeutung zugemessen werden, da im OP ein grosser Teil der Wertschöpfung erbracht wird. Viele Experten sind sich einig, dass die Ernennung eines formellen OP-Managers zur Steigerung der OP-Effizienz entscheidend ist [11-13]. Allerdings sollte ein OP-Manager mit entsprechenden Entscheidungsbefugnissen ausgestattet sein, um seine Entscheidungen effektiv umzusetzen. Seine Position sollte innerhalb der Spitalstruktur relativ hoch angesiedelt sein, um die strategische Bedeutung des OP innerhalb eines Spitals zu betonen. OP-Manager werden zunehmend Controllinginstrumente anwenden müssen, um die Effektivität ihrer operativen Entscheidungen und Strategien messen und ihre Aktivitäten entsprechend anpassen zu können [14].

Nebst der Institution eines OP-Managers ist die Erstellung klar verständlicher und verbindlicher Richtlinien von grosser Wichtigkeit [12]. Nach Waisel scheinen sich im OP Gefangenendilemmasituationen oft $\mathrm{zu}$ wiederholen, bei welchen Angestellte entweder miteinander kooperieren oder aber aufgrund unterschiedlicher Interessen sich gegenseitig behindern [15]. Im
Konflikt können Lösungen entstehen, die oft zu Lasten der einen Partei oder des Patienten gehen und somit sowohl die Prozess- als auch die Outcome-Qualität negativ beeinflussen (z.B. Wartedauer, medizinische Behandlungsqualität). Das Ziel ist es, eine Kultur mit Vertrauen, Respekt, guter Kommunikation und einer positiven Erwartungshaltung zu schaffen. Das Bewusstsein, dass die Entstehung dieses «sozialen Kapitals» jedermanns Verantwortung ist, soll gefördert werden. Erfolgreiche Interaktionen sollen gefördert werden durch die Realisierung transparenter, funktionierender und allgemeingültiger Richtlinien, die auf den Werten der Organisation basieren.

In den letzten Jahren wurden in den USA zahlreiche Erkenntnisse im Forschungsbereich der operativen Entscheidungsfindung im OP-Management gewonnen. In zahlreichen Arbeiten konnte gezeigt werden, dass statistisch-mathematische Methoden den herkömmlichen, deterministischen Verfahren im Hinblick auf die Maximierung der OP-Effizienz (bzw. Minimierung der Ineffizienz) deutlich überlegen sind. In einer kürzlich erschienenen Arbeit von Freytag et al. wurde gezeigt, wie sich eine solch rationale Betriebsführung auf ein deutsches Krankenhaus übertragen lässt [16]. Das Grundprinzip der statistisch-mathematischen Methode zur Maximierung der OP-Effizienz beruht auf der Erkenntnis, dass nur die Minimierung von Unter- und Übernutzungskosten (durch unbeschäftigtes Personal respektive Überstunden) zur Maximierung der OP-Effizienz beitragen. Erstaunlicherweise ist das verwendete mathematische Modell in erster Linie auf Patientensicherheit begründet, in zweiter Linie darauf, dass Chirurgen an jedem beliebigen Arbeitstag operieren können und erst in dritter Linie auf der Maximierung der OP-Effizienz. Studien, die Instrumente zur Minimierung der Ineffizienz am Operationstag analysieren, können auf die Schweizer Situation problemlos umgesetzt werden [17].

Die Praxis der überlappenden Ein-/Ausleitung, die in der Schweiz vielerorts traditionsgemäss zu finden ist, muss neu überdacht werden. Der Nutzen des parallelen Arbeitens ist bei wenigen langen Eingriffen viel kleiner als bei vielen kleinen Eingriffen, bei denen sich die Allokation eines zweiten Anästhesieteams positiv auf die Effizienz auswirkt [18]. Beispielsweise macht nach dem deutschen Bundesangestelltentarif und bei fixen Kernarbeitszeiten überlappendes Einleiten erst dann Sinn, wenn die Eingriffe ein relatives Fallgewicht von >1,33 aufweisen. Allerdings ist hier die Vergleichbarkeit von Studienresultaten nicht 1:1 auf jedes beliebige Kranken- 
haus übertragbar, sondern jeder OP-Manager wird die Berechnung von Kosteneinsparungen durch paralleles Arbeiten basierend auf den zu erwartenden Deckungsbeiträgen selber durchführen müssen.

\section{Fall-/Prozesskostenrechnung, Kostenanalysen}

Jedes Spital wird seine eigenen internen Fall- und Prozesskostenrechnungen durchführen müssen, um die Fallkosten den auf den DRG-Codes basierenden Erlösen gegenüberstellen zu können.

Anhand einer in Deutschland durchgeführten retrospektiven Kostenanalyse haben Schuster et al. die wichtigsten anästhesiebedingten Kostenkomponenten untersucht [19]. Dabei hat sich gezeigt, dass Unterschiede in der Anästhesiedauer den wichtigsten Kostentreiber darstellt, während andere Faktoren (elektiver Eingriff versus Notfall, ASA Status $\geq 3$, extreme Patientenalter, Legen von invasiven Kathetern) wenig zur Erklärung von Kostenunterschieden zwischen den Subspezialitäten beitragen konnten. Personal- und Gesamtkosten waren am höchsten bei Subspezialitäten mit den kürzesten Eingriffszeiten, d.h. Anästhesiekosten pro Minute fallen mit steigender Eingriffsdauer aufgrund vorhandener Fixkosten pro Fall.

Kostenanalysen, die auf amerikanischen Studienresultaten basieren, müssen mit Besonnenheit interpretiert werden. Beispielsweise hat eine Untersuchung von Deckungsbeiträgen pro OPStunde gezeigt, dass diese sowohl zwischen Chirurgen und auch zwischen verschiedenen Disziplinen stark variiert [20]. Für die privaten amerikanischen Spitäler war diese Erkenntnis damals neu und stellte eine grosse Hilfe für lang-

\section{Abbildung 2}

Komplexität der Behandlungsprozesse. In Anlehnung an eine Abbildung im Buch OP-Management der Medizinisch Wissenschaftlichen Verlagsgesellschaft, Berlin. Mit freundlicher Genehmigung des Verlages.

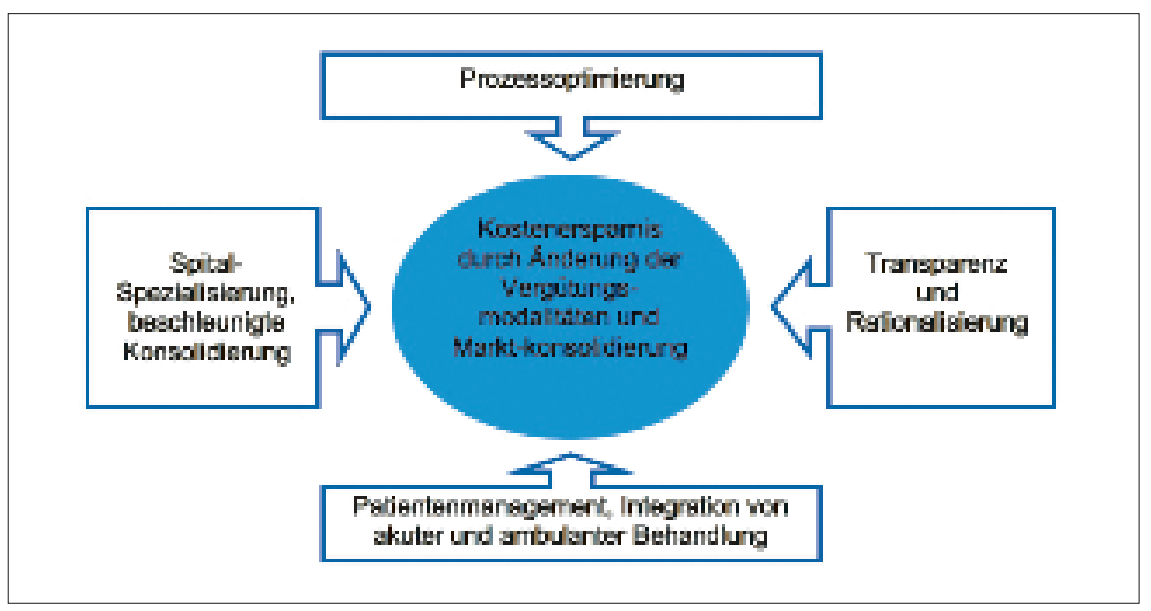

fristige, strategische Entscheidungen dar. Üblicherweise wurde vorher die OP-Benutzungsrate und nicht der Deckungsbeitrag als Endpunkt für die OP-Bewirtschaftung genommen, was sich aber mit dieser Studie als wirtschaftlich ungünstig erwies. Die Erkenntnisse dieser Studie mögen zwar für Betreiber von privaten, chirurgischen Zentren in der Schweiz äusserst nützlich sein, sind allerdings für OP-Manager im öffentlichen Spital nicht direkt umsetzbar. Denn typischerweise sind in Schweizer Spitälern OP-Betriebe als Kostenstellen organisiert und arbeiten nicht wie eigenständige Betriebe mit Deckungsbeiträgen. Aus diesem Grund müsste für die Schweizer Verhältnisse die Interpretation einer solchen Studie zuerst angepasst werden, wobei das Ziel nicht die Maximierung der Deckungsbeiträge wäre, sondern die Minimierung der variablen Kosten.

Heller et al. untersuchten in ihrer Arbeit anhand einer Prozesskostenanalyse die betriebswirtschaftlichen Auswirkungen der thorakalen Epiduralanästhesie bei urologischen Operationen [21]. Dabei zeigte sich, dass sich die Modifikation des Behandlungspfades positiv auf das Kosten-Gewinn-Verhältnis (Deckungsbeitrag) einer DRG-Gruppe auswirken kann.

\section{Schlussfolgerungen / Handlungsbedarf (Abb. 2)}

Das DRG-System mit seinen neuen wirtschaftlichen Anreizen wird die Komplexität der Behandlungsprozesse noch weiter verstärken. Sowohl für die einzelnen Abteilungen als auch für die Mitarbeiter stellt die konsequente Orientierung auf integrierte Behandlungspfade in Zukunft den Schlüssel zum Erfolg dar. Die Spannung zwischen den zwei häufig entgegengesetzten Zielen, nämlich zwischen dem Wohlergehen des Patienten und dem finanziellen Wohlergehen des Betriebes, lässt sich nur mit einem konsequent auf den Behandlungsprozess ausgerichteten Vorgehen abbauen. Innerhalb der Abteilungen wird der Behandlungsprozess, abgeleitet von internen Richtlinien und ausgearbeitet von beteiligten Berufsgruppen, zum zentralen Instrument, an welchem die entstehenden Kosten gemessen werden können. Innerhalb des OP müssen tiefgreifende Restrukturierungen in Abhängigkeit bestehender Strukturen erfolgen, und Standard Operating Procedures führen zu effektiveren und effizienteren Abläufen. Die Koordination der beteiligten Berufsgruppen und die Entwicklung einer Unternehmenskultur im Sinne des Social Capital stellen grundlegende Herausforderungen an den OP-Manager dar.

Die Kenntnisse der internen Kosten sind insbesondere in bezug auf eine allfällige Neuaus- 
richtung des Behandlungsangebotes von grosser Wichtigkeit. Mit Einführung der SwissDRG wird auf der einen Seite Preistransparenz für stationäre Leistungen geschaffen, auf der anderen Seite sind die Leistungserbringer zunehmend gezwungen, kosteneffizient und mit hoher Ergebnisqualität $\mathrm{zu}$ arbeiten. Um dies zu erreichen, werden sich die Spitäler zunehmend spezialisieren, Kompetenzen aufbauen und entsprechende Geschäftsfelder ausbauen. Die Einführung der SwissDRG verlangt von den Spitälern die Übernahme von Verantwortung auf allen Führungsebenen, den Einbezug aller Mitarbeiter und das konsequente wirtschaftliche Ausrichten aller Behandlungsprozesse. Die Einführung der SwissDRG wird die Spitallandschaft Schweiz verändern. Die Herausforderung liegt nun darin, die positiven Aspekte der Kosten-, Leistungs- und Prozesstransparenz zu optimieren und gleichzeitig eine hohe Behandlungsqualität zu garantieren. Letztere Definition ist allerdings auch schwierig.

\section{Literatur}

1 SwissDRG. Pressemitteilung vom 19. Dezember 2005. Verein SwissDRG; 2005.

2 SwissDRG. Newsletter Nummer 4, 2005.

3 Bauer M, Hanss R, Schleppers A, Steinfath M, Tonner PH, Martin J. Prozessoptimierung im «kranken Haus». Anaesthesist 2004;53(5):414-26.

4 Bach A. Anforderungen an das System OP Organisatorische Voraussetzungen/Kosten. Zentralbl Chir 2001;126(12):336.

5 Reith HB. Welche Auswirkungen wird die Einführung von G-DRGs auf chirurgische Abteilungen haben? Zentralbl Chir 2002;127(8):651-5.

6 Tischendorf JJ, Crede S, Herrmann P, Bach N, Bomeke C, Manns MP, et al. Diagnosenverschlüsselung durch Medizinische Dokumentationsassistentin oder Stationsarzt. Einfluss auf die Abbildung der DRG-Leistung. Dtsch Med Wochenschr 2004;129(33):1731-5.

7 Roeder N, Hensen P, Hindle D, Loskamp N, Lakomek HJ. Instrumente zur Behandlungsoptimierung. Klinische Behandlungspfade. Chirurg 2003;74(12):1149-55
8 Lauterbach KW, Schrappe M. Gesundheitsökonomie, Qualitätsmanagement und Evidence-based Medicine. 2. Ausgabe. Stuttgart: Schattauer; 2004.

9 Müller H, Schmid K, Conen D. Interne Leitlinien und Patientenpfade. Med Klin 2001;96(11):692-7.

10 Laux T, Mock H, Madler C. Was ist Anästhesiologie in den G-DRGs wert? Erste Erfahrungen mit den G-DRGs. Anaesthesiol Reanim 2004;29(3):79-86.

11 Bach A, Bauer M. Ökonomische Aspekte in der Anästhesie. Teil I: Rahmenbedingungen in der Bundesrepublik Deutschland. Anasthesiol Intensivmed Notfallmed Schmerzther 1998; 33(3):135-49.

12 Geldner G, Eberhart LHJ, Ruoff M, Trunk S, Dahmen KG, Reissmann T, et al. Effizientes OP-Management. Vorschläge zur Optimierung von Prozessabläufen als Grundlage für die Erstellung eines OP-Statuts. Gynaekologe 2003;36:149-57.

13 Gebhard E, Hartwig E, Isenmann R, Triebsch K, Gerstner H, Bailer M, et al. OP-Management: Chirurg oder Anästhesist? Anaesthesist 2003; 52(11):1062-7.

14 Schüpfer G, Bauer M, Scherzinger B, Schleppers A. Controllinginstrumente für OP-Manager. Anaesthesist 2005;54(8):800-7.

15 Waisel DB. Developing social capital in the operating room: the use of population-based techniques. Anesthesiology 2005;103(6):1305-10.

16 Freytag S, Dexter F, Epstein RH, Kugler C, Schnettler R. Zuweisung und Planung von Operationskapazitäten. Chirurg 2005;76(1):71-9.

17 www.franklindexter.net.

18 Hanss R, Buttgereit B, Tonner PH, Bein B, Schleppers A, Steinfath M, et al. Overlapping induction of anesthesia: an analysis of benefits and costs. Anesthesiology 2005;103(2):391-400.

19 Schuster M, Standl T, Wagner JA, Berger J, Reimann H, Am Esch JS. Effect of different cost drivers on cost per anesthesia minute in different anesthesia subspecialties. Anesthesiology 2004; 101(6):1435-43.

20 Macario A, Dexter F, Traub RD. Hospital profitability per hour of operating room time can vary among surgeons. Anesth Analg 2001;93(3):669-75.

21 Heller AR, Litz RJ, Wiessner D, Dammann C, Weissgerber R, Hakenberg OW, et al. Betriebswirtschaftliche Auswirkungen der thorakalen Epiduralanästhesie bei urologischen Operationen. Anaesthesist 2005;54(12):1176-85. 